\title{
IBM PC, PS/2, and compatibles
}

\author{
DRAKE R. BRADLEY \\ Bates College, Lewiston, Maine
}

A total of eight people met for over $2 \mathrm{~h}$ to discuss the use of PCs for experimentation, teaching, and other activities. Several of the more important issues that were raised are summarized below.

\section{Automated Test Generation}

It was pointed out that many of the test banks and testgeneration programs supplied by publishers are poorly designed. There was general consensus on the criteria defining a good test-generation program: (1) It should be available to instructors whether or not they use the associated textbook; (2) it should allow either random or nonrandom subsamples of questions to be selected from the test bank and collated in a random order, along with a matching key; (3) it should allow the instructor to supplement the test bank with his or her own questions; and (4) it should permit easy editing of the questions in the test bank. It was suggested that a common format for storing questions in a test bank should be adopted. In that case, test-generation software with a high degree of flexibility and power could be developed and marketed independently of any particular publisher's test bank. From the instructor's viewpoint, this approach would be far superior to the current situation, in which each publisher supplies test-generation programs having varying capabilities as well as incompatible formats for the test-bank questions.

Two tricks using WordPerfect were mentioned that are useful to instructors maintaining their own test banks. One is to define each test question as a numbered footnote. Then, as questions are added or deleted, WordPerfect will automatically renumber the questions in the right sequence. The second trick allows the instructor to randomly select a subsample of items from a test bank. This involves generating a random sequence of digits from $1-N$, using a separate program, and then copying these into one field of a WordPerfect file. The WordPerfect SORT command may then be used to sort the questions based on the random numbers. Taking the first $n$ items from the sorted list constitutes the desired random sample of test questions.

\section{Software for Courses}

It was pointed out that the policies for obtaining software vary greatly from publisher to publisher. In some cases, software is provided free with adoption of a text-

Correspondence may be sent to Drake R. Bradley, Department of Psychology, Bates College, Lewiston, ME 04240. book, and in other cases, it is purchased separately. In the latter case, the wide variety of pricing and licensing arrangements that exist make it difficult to assess the relative values of different software packages. It was argued that since publishers use software primarily to sell textbooks, they are failing to respond adequately to the separate and legitimate need in the academic market for software designed to support instruction.

\section{Menu- versus Command-Driven Software}

The relative advantages and disadvantages of menuand command-driven software packages were discussed. There was general consensus that menu-driven systems were easier for students to learn and use. However, as expertise is acquired, users often come to prefer commanddriven systems, because such systems do not require descending and ascending through the multiple levels of a menu. A distinction was also drawn between "user friendly" and "easy-to-use" software: The former refers to software that novices can begin using immediately, and that provides extensive guidance and hand-holding along the way; the latter refers to software that may require a manual to use initially, but that is designed so that it is extremely easy to accomplish the tasks desired by the user (e.g., complex statistical analyses).

It was tacitly acknowledged that our colleagues attending the Macintosh User Group meeting in the adjacent room were strong advocates of user-friendly, menu-driven systems. Someone joked that the Mac users would probably have greater difficulty finding their meeting room because the SCIP Program did not provide a menu with a point-and-click option. Sure enough, about $10 \mathrm{~min}$ later, an individual wandered into the room and asked if this was the Mac User Group meeting! All of which goes to show that while PC/MC-DOS users might be dull and boring, they are not without a sense of humor.

On a more serious note, some concern was expressed over the continuing division of the computer world into two distinct groups: Macintosh and IBM PC users. The problems with marketing and acquiring software across machines were mentioned. It was noted that in the next generation of machines, this division should be less obvious, and that such machines should be capable of emulating either the Macintosh or the IBM PC.

\section{Super Cartridges for the HP}

Super cartridges for the HP laser printer are now available for around $\$ 600$ that combine all of the fonts avail- 
able on the various individual cartridges provided by HP. This provides a cost-effective alternative to purchasing many different cartridges (only some of which can be loaded at a given time), or to installing and downloading soft fonts from disk. It was noted that WordPerfect 5.1 has an equation editor, which will be very useful to authors needing to include mathematical equations in their documents.

\section{Developments in Pricing}

Lotus has implemented new educational prices for any of their products sold through college and university bookstores. For example, Version 2 of Lotus 1-2-3 will be available for around $\$ 99$, and Version 3 for around $\$ 129$.

\section{Improvements in MS-DOS}

The question was raised as to whether any attempt to improve MS-DOS would be made. Although new and better operating systems for IBM PCs are coming, they require more memory than is available for most of the PCs currently in place. The absence of a good command processing language (CPL) for MS-DOS was noted.

\section{Administrative versus Academic Computing}

The many trials and tribulations of doing academic and administrative computing on the same machine were discussed. Examples of academic computing being curtailed in order to complete "deadline" administrative comput- ing, such as payroll, were cited. However, one participant noted that academic and administrative computing had been successfully integrated at his university, and that this greatly facilitated the exchange of information (course enrollment lists, grades, schedules, etc.) between the two groups. With greater reliance being placed on distributed processing, as in using microcomputers and/or mainframes linked to a common network, the issue of academic as opposed to administrative computing is becoming less critical.

\section{Software "Poster" Sessions at SCIP}

It was suggested that future meetings of SCIP might want to sponsor a software "poster" session. SCIP members would submit a proposal to demonstrate their software on microcomputers at some prearranged time, much as Psychonomic Society members arrange for "poster" sessions at one of several times allocated during that meeting. Implementation of this idea would require finding vendors to supply Macintosh and IBM-compatible PCs for the session. In contrast with paper presentations, the software poster sessions would allow SCIP participants to interact directly with the software being demonstrated. In addition, public domain (nonproprietary) software could be copied by participants for use in their own courses and laboratories. In this way, the software "poster" session would serve the ancillary function of being a software exchange program. 